\title{
Review of: "Spiderweb Nanomechanical Resonators via Bayesian Optimization: Inspired by Nature and Guided by Machine Learning (Adv. Mater. 3/2022)"
}

Dai Yanning ${ }^{1}$

1 Beijing University of Aeronautics and Astronautics

Potential competing interests: The author(s) declared that no potential competing interests exist.

The present study presents a novel Bayesian optimization-based technique for nanomechanical resonators design. As a result of the numerical simulation, it was observed that, starting with a basic spiderweb architecture, the optimized result displays high consistency with the spiderweb vibration mechanisms in nature. The device performance achieves a high quality factor of above 1 billion in room-temperature environments and is verified by experiments. This study is interesting and makes an important contribution to the literature on the fusion of human intuition and artificial intelligent algorithms. It lays the foundation for future studies to discover diverse strategies for metamaterial and device design. 